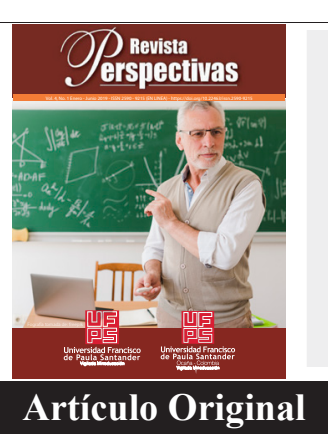

\title{
Conocimiento y práctica pedagógica de los docentes en escolares con inatención, hiperactividad e impulsividad
}

Knowledge and pedagogical practice of teachers in front of schoolchildren with inattention, hyperactivity and impulsiveness

Conhecimento e prática pedagógica dos professores em crianças em idade escolar com desatenção, hiperatividade e impulsividade

Diego Rivera-Porras $^{\mathrm{a}^{*}}$, Sandra Milena Carrillo-Sierra ${ }^{\mathrm{b}}$, Gloria Silva-Monsalve ${ }^{\mathrm{c}}$, Laura Nataly Galvis-Velandia ${ }^{\mathrm{d}}$

$a^{*}$ Maestría en gerencia de recursos humanos, die.rivera@mail.udes.edu.co, ORCID 0000-0003-2169-3208, Universidad de Santander, Cúcuta, Colombia.

${ }^{b}$ Maestría en desarrollo educativo y social, sa.carrillo@mail.udes.edu.co, ORCID 0000-0001-9848-2367, Universidad de Santander, Cúcuta, Colombia.

cEspecialización en pedagogía para la docencia universitaria, coord.psicologia@cucuta.udes, ORCID 0000-0002-9517-4163, Universidad de Santander, Cúcuta, Colombia.

Maestría en educación, lauranatalygv@ufps.edu.co, ORCID 0000-0003-2444-8398, Universidad Francisco de Paula de Santander, Cúcuta, Colombia.

Cómo citar: Rivera, D., Carrillo, S. M., Silva, G. \& Galvis, L. N. (2019). Conocimiento y práctica pedagógica de los docentes en escolares con inatención, hiperactividad e impulsividad. Perspectivas, 4(1), 66-76.

Recibido: Agosto 10, 2018; Aceptado: Noviembre 15, 2018

\begin{tabular}{ll}
\hline RESUMEN \\
\hline Palabras Clave: & $\begin{array}{l}\text { Esta investigación tuvo como objetivo describir el conocimiento y la práctica pedagógica de los docentes en escolares } \\
\text { con inatención, hiperactividad e impulsividad en un colegio de la ciudad de Cúcuta. Este estudio se realizó bajo una } \\
\text { metodología de investigación cuantitativa con diseño no experimental con alcance descriptivo y de corte transversal. Se }\end{array}$ \\
Didáctica & utilizó la Escala Likert de Conocimiento y Práctica Pedagógica - CPIHI conformada por las dimensiones de Inatención, \\
Hiperactividad & Hiperactividad, Impulsividad, Relación Pedagógica y Didáctica, dicho instrumento cuenta con una Confiabilidad de 0,82 \\
Impulsividad & y una consistencia interna del $95 \%$ entre sus dimensiones. El instrumento se aplicó a 27 docentes de un Colegio Público \\
Inatención & y se encontró como resultado que a mayor conocimiento sobre la inatención, hiperactividad e impulsividad, mejor es la \\
Práctica pedagógica & relación y la didáctica en la práctica pedagógica. \\
Relación pedagógica &
\end{tabular}

\begin{tabular}{ll}
\hline & ABSTRACT \\
\hline Keywords: & This research aimed to describe the knowledge and pedagogical practice of teachers in students with inattention, \\
& hyperactivity and impulsivity in a school in the city of Cúcuta. This study was carried out under a quantitative research \\
methodology with a non-experimental design with a descriptive and cross-sectional scope. We used the Pedagogical & Knowledge and Practice Method - CPIHI formed by the dimensions of Intention, Hyperactivity, Impulsivity, Pedagogical \\
Didactics & and Didactic Relationship, this instrument has a Reliability of 0.82 and an internal consistency of $95 \%$ among its \\
Hyperactivity & dimensions. The instrument was applied to 27 teachers of a Public School and it was found that the more knowledge about \\
Impulsivity & inattention, hyperactivity and impulsivity, the better the relationship and the didactic in pedagogical practice.
\end{tabular}

Pedagogical relationship

\begin{tabular}{ll}
\hline & RESUMO \\
\hline Palavras chave: & O objetivo desta pesquisa foi descrever o conhecimento e a prática pedagógica de professores em escolares \\
& desatentos, hiperativos e impulsivos de uma escola da cidade de Cúcuta. Este estudo foi realizado no âmbito \\
Didáctica & de uma metodologia de investigação quantitativa com desenho não experimental de âmbito descritivo e corte \\
Hiperactividade & transversal. Foi utilizada a Escala Likert de Conhecimento e Prática Pedagógica - CPIHI, composta pelas \\
Impulsividade & dimensões de Desatenção, Hiperatividade, Impulsividade, Relação Pedagógica e Didática. Este instrumento \\
Desatenção & possui uma Confiabilidade de 0,82 e uma consistência interna de $95 \%$ entre suas dimensões. O instrumento foi \\
Prática pedagógica & aplicado a 27 professores de uma Escola Pública e verificou-se como resultado que, quanto maior o conhecimento \\
Relação pedagógica & sobre desatenção, hiperatividade e impulsividade, melhor o relacionamento e a didática na prática pedagógica.
\end{tabular}

\section{Introducción}

El presente artículo recoge los resultados de un estudio, cuya finalidad ha sido determinar los conocimientos y prácticas de los docentes hacia la inclusión de estudiantes con algunas necesidades educativas especiales como el déficit de atención, hiperactividad e impulsividad. Siendo las practicas pedagógicas una herramienta clave frente la generación de conocimiento y acciones legislativas de la educación en el país (Aperador, Díaz y Orozco, 2015; Cantor-Silva, Pérez-Suarez y CarrilloSierra, 2018; Hurtado-Olaya, García-Echeverri, Rivera-Porras y Forgiony-Santos, 2018; Forgiony-Santos, 2019), donde las

*Autor de Correspondencia.

E-mail: die.rivera@mail.udes.edu.co (Diego Rivera Porras)

(c) (i) $\Theta$ Peer review is the responsibility of the Universidad Francisco de Paula Santander.

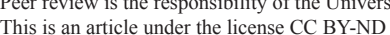


connotaciones de inclusión para favorecer los niños, niñas y adolescentes que presentan estas características específicas en el aula (Costa, Barros, Lopes y Marques, 2015), las cuales se han convertido en un factor relevante y de creciente atención para las instituciones de educación, directivos, docentes y acudientes o padres de familia (Davini, 2015).

Respecto al tipo de diseño, se trata de un estudio descriptivo de tipo trasversal (López y Fachelli, 2015). La aplicación fue realizada concretamente en un centro educativo, de orden público, de educación secundaria obligatoria de la ciudad de Cúcuta. La selección de los docentes se realizó por un muestreo de conveniencia, con criterios de inclusión (Cauas, 2015). El instrumento utilizado para la recolección de la información fue la Escala Likert de Conocimiento y Práctica Pedagógica de los Docentes frente a los Escolares con Inatención, Hiperactividad e Impulsividad - CPIHI, la cual está conformada por un total de 32 reactivos, divididos en 2 variables, la primera es Conocimiento y está conformada por 3 dimensiones (Inatención: 8 reactivos, Hiperactividad: 4 reactivos e Impulsividad: 3 reactivos) y la segunda variable denominada Práctica Pedagógica está conformada por 2 dimensiones (Relación Pedagógica: 8 reactivos y Didáctica: 9 reactivos).

Los resultados señalan, por un lado, que de manera general los docentes poseen actitudes positivas que le permiten desarrollar mayores posibilidades de interacción con el estudiante (Bazán y González, 2017; Bonilla-Cruz, VillamizarBermúdez y Arenas-Villamizar, 2017; Arenas-Villamizar, Silva-Monsalve, Annicchiárico-Lobo, Contreras-Manrique, Luna-Rodríguez y Raynaud, 2018), esta condición se presenta como una serie de factores importantes para desarrollar buenas prácticas educativas (De Tezanos, 2015), frente a lo cual esto se podría considerar un avance significativo en el ambiente educativo para el fomento de políticas y culturas inclusivas entre las redes de centros educativos que cuenten con apoyo para tal fin (Echeverry, 2017).

Por otra parte, se evidenció que la formación, dominio y adquisición de conocimientos es un elemento proporcional y correspondiente a las necesidades del medio académico y de la educación, que a su vez exige una adaptación ante aquellas realidades complejas y cambiantes (MontánchezTorres, Jornet-Meliá, Perales-Montolío, Carrillo-Sierra y Wilches-Durán, 2017; Bonilla-Cruz, Forgiony-Santos, Hernández-Lalinde, Carrillo-Sierra, Rivera-Porras, NuvánHurtado y Martínez-Santana, 2018). Las herramientas con las que cuenta un docente para responder a la diversidad de conductas trascienden los requerimientos físicos y espaciales (Armijos, 2017); es decir, estos son elementos importantes que acompañan el ejercicio de la docencia en la enseñanza, y siendo un factor diferencial de estas prácticas, podría estar asociado a la autonomía del docente, a su forma de concebir el ejercicio de la docencia (conocimientos), a la creatividad en contexto (prácticas que desarrolla en el aula) (Estévez y León,
2014); lo cual le permite tomar de decisiones frente a la forma en que se puede llegar desempeñar con en el estudiante en el proceso de aprendizaje, es decir, frente a cómo logra orientar una asignatura ante la particularidad cognitiva y conductual de un estudiante (Henao, Isaza y Gómez, 2017; Carrillo-Sierra, Sanabria-Herrera, Bermúdez-Pirela y Espinosa-Castro, 2018; Carrillo-Sierra, Rivera-Porras, Forgiony-Santos, NuvánHurtado, Bonilla-Cruz y Arenas-Villamizar, 2018).

\section{Bases teóricas}

En Colombia se visualiza la administración de un establecimiento escolar a partir de cuatro áreas de gestión: directiva, académica, administrativa y financiera $y$ de gestión de la comunidad (Garzón, 2017). Las áreas, de igual forma, contienen procesos básicos definidos y estos a su vez componentes que encaminan la acción administrativa y docente. Según las pautas para la gestión académica sugeridas por el Estado, lo que se hace en el aula de clases para colaborar con el aprendizaje del otro es potenciar la relación pedagógica y formativa (Torres, 2015); por tanto dicha formación obedece a criterios de desarrollo social e individual, en los cuales se integran los pilares básicos de la educación y que hacen referencia a aprender a conocer, es decir, adquirir los instrumentos de la comprensión (Palomares, 2017); aprender a hacer, para poder influir sobre el propio entorno; aprender a vivir juntos, para participar y cooperar con los demás en todas las actividades humanas; por último, aprender a ser (Delors, 2013; Calderón, 2016; Duque-Moncada, Quintero-Sánchez, Remolina-Corredor, Carrillo-Sierra y Rivera-Porras, 2018; Angarita-Ortiz, Forgiony-Santos y Rivera-Porras, 2018).

Por tanto, la educación escolar obedece a estándares de desempeño integrales recíprocos que busquen la mejora continua de los agentes activos del procesos educativo, para lo cual, los docentes deben contar con estándares de desempeño en el aula, curriculares y de gestión escolar ( González-Gómez y Carrillo-Sierra, 2018; Rivera-Porras, Gallardo-Pérez, MoraOrtiz, Gómez-Machado y Barrios-Barrios, 2018; VillamizarNovoa, Rodríguez-Viloria, Carrillo-Sierra y Torrado, 2018); que se relacionan directamente con el mejoramiento continuo de los procesos de enseñanza - aprendizaje (Centro de Estudios Educativos y Servicios Integrales de Evaluación y Medición Educativa (SIEME) y Heurística Educativa, 2013).

Estos estándares incluyen las prácticas pedagógicas, integradas como un proceso perteneciente al área de gestión académica de la institución y que se identifica como una "experiencia antropológica de cualquier cultura, aquella que se desprenden de la propia institucionalización de la educación en el sistema escolar y dentro del marco en el que se regula la educación" (); se relaciona con la organización de "las actividades de la institución educativa para lograr que los estudiantes aprendan y desarrollen sus competencias" (Gimeno, 1997; Diker, 1997 citados por Moreno, 2017) 
cuyos componentes básicos se pueden esbozar a través de la diversidad didáctica para las áreas de conocimiento, asignaturas y proyectos transversales, estrategias para el desarrollo de las actividades y tareas escolares, uso articulado de los recursos y los tiempos para el aprendizaje (Calvo, Lara y García, 2017). Por lo tanto, el conocimiento como apoyo de la práctica pedagógica, fortalece la buena docencia, la cual se adhiere a estándares altos relativos a contenido y forma de enseñar. Más específicamente, la buena docencia está en armonía con principios moral y racionalmente defendibles (Preiss, Calcagni, Espinoza, Gómez, Grau, Guzmán, Müller, Ramírez y Volante, 2014).

Entonces, las prácticas de los docentes permiten materializar aspectos ligados a los objetivos legislativos y políticos de un país, existen documentos en los que se proponen estrategias metodológicas y actividades didácticas que muestran una coherencia en cuanto al contexto donde se desarrollan, las cuales deben ser asumidas a nivel país. Así, las prácticas pedagógicas se abordan inicialmente a partir de un marco de referencia del contexto sociocultural, desde las particularidades y necesidades de una ciudad o región y diferenciando el objetivo clave tanto de la educación como de la didáctica, ya que la primera se relaciona al cómo educar y la segunda al cómo enseñar (Lucio, 2017).

De igual manera la mirada a las prácticas de los docentes implica una segunda perspectiva, asociada el componente pragmático, cognitivo, actitudinal y aptitudinal, desde su postura o concepción del ejercicio de la docencia en términos de propósitos formativos y la relación con su estudiante hasta como estas estructura contenidos, materiales, actividades y metodologías están ligadas, sobre todo para la atención, a las particularidades de estudiantes que oscila desde la diversidad cognitiva, conductual y emocional, hasta la discapacidad en el aula (Estévez, 2015).

A partir de eso, la distinción de criterios específicos frente a las características del alumnado en temas de impulsividad, inatención e hiperactividad, es la que fundamenta el accionar pedagógico del docente, en cuanto este orienta los procesos de inclusión, enseñanza y mitigación de efectos adversos para el desempeño dentro del aula; de este modo, se establece que la existencia de una evaluación psicopedagógica es imprescindible, puesto que con ella se puede valorar las dificultades relacionales, afectivas, académicas, es decir, las necesidades psicoeducativas derivadas, con el fin de establecer objetivos de intervención psicoeducativa que amortigüen los síntomas característicos del trastorno (Gómez, Soria, Rico y Carrasco, 2017).

Es por ello que la funcionalidad interdisciplinar de la pedagogía y la didáctica, cumplen un objetivo armónico en el que se enfatizan tanto los lineamientos formativos como las metodologías empleadas en el aula por parte del docente, en cuanto al mejoramiento de la enseñanza impartida; más específicamente se menciona que la pedagogía se dirige a la orientación de la labor del educador y la didáctica por su parte, se encarga de su labor como docente (Lucio, 2017).

Al margen de lo que los docentes puedan desconocer, los horizontes conceptuales de la política de educación inclusiva están claros, por lo menos para los organismos internacionales y el Estado (Acosta, 2016). Lo anterior se ve reflejado en los intereses de los agentes de salud dirigido hacia la modificación de comportamientos desde una lógica preventiva, central en el modelo biomédico y los contenidos definidos por las instituciones de salud, los cuales aplican a su vez en el escenario educativo, propiamente en las prácticas pedagógicas y relaciones docente estudiante en las cuales el "control y el dominio" se encuentran en el educador (Davini, 2015). Entonces, un grupo -los agentes de salud- tiene el poder para definir los significados legítimos sobre la crianza, la educación tras lo cual se produce un "control simbólico", es más, al relacionar las prácticas pedagógicas y la educación inclusiva aparecen propuestas que intentan la construcción de una escuela no discapacitante, por ejemplo, el llamado diseño universal de aprendizaje.

\section{Materiales y métodos}

Este estudio se realizó bajo la metodología de investigación cuantitativa con diseño no experimental, con alcance descriptivo y corte transversal, ya que se recolectaron y analizaron datos de las variables estudiadas (Cauas, 2015). Este tipo de investigación aplica modelos matemáticos, teorías e hipótesis relacionados con el problema y emplea el método científico centrado en aspectos observables y susceptibles de cuantificación, también usa la estadística para el análisis de los datos de manera científica (Rivero, 2013). En este tipo de investigación no existen condiciones, ni estímulos provocados o dirigidos, es decir, los sujetos son observados en su ambiente natural y se analiza la forma en que se presentan los hechos en un momento único en tiempo y espacio, buscando especificar las características de las variables y de las dimensiones medidas (Pérez, Molina y Lechuga, 2013).

Para recolectar la información se realizó un muestreo por conveniencia, ya que es una técnica comúnmente usada, que consiste en seleccionar una muestra de la población por el hecho de que sea accesible (Rivero, 2013). Así mismo, se tuvieron en cuenta criterios de inclusión como pertenecer la institución educativa, participar voluntariamente de la investigación y el diligenciamiento del consentimiento informado. La población estuvo conformada por 30 docentes y la muestra por un total de 27 docentes de un Colegio Público de Cúcuta - Norte de Santander.

\section{Instrumento}

Se utilizó la Escala Likert de Conocimiento y Práctica Pedagógica de los Docentes frente a los Escolares con 
Inatención, Hiperactividad e Impulsividad - CPIHI, la cual mide de forma objetiva fenómenos en torno a las actitudes de los individuos. El instrumento presenta opciones de respuesta graduales que van desde Nunca (1), Casi nunca (2), A veces (3), Casi siempre (4) y Siempre (5) y está conformado por un total de 32 reactivos, divididos en 2 variables, la primera es Conocimiento y está conformada por 3 dimensiones (Inatención: 8 reactivos, Hiperactividad: 4 reactivos e Impulsividad: 3 reactivos) y la segunda es Práctica Pedagógica y está conformada por 2 dimensiones (Relación Pedagógica: 8 reactivos y Didáctica: 9 reactivos).

\section{Propiedades psicométricas de la escala.}

Confiabilidad. Alfa de Cronbach 0,82 basado en las dimensiones de la escala (5 elementos). Alfa de Cronbach por dimensiones: Inatención $(\alpha: 0,76)$, Hiperactividad $(\alpha$ : $0,77)$, Impulsividad $(\alpha: 0,79)$, Relación Pedagógica $(\alpha: 0,77)$ y Didáctica $(\alpha: 0,82)$.

Consistencia Interna. Correlación de Spearman: Inatención e Hiperactividad ( $p$ 0,00), Inatención e Impulsividad ( $p 0,00$ ), Inatención y Relación Pedagógica ( $p$ $0,01)$, Hiperactividad e Impulsividad (p 0,02), Hiperactividad y Relación Pedagógica (p 0,00), Impulsividad y Relación Pedagógica (p 0,01), Impulsividad y Didáctica (p 0,002), Relación Pedagógica y Didáctica (p 0,00).

Validez. Índice Kaiser-Meyer-Olkin: 0,71. Test de Esfericidad de Bartlett: 48,97 (p 0,00). Análisis Factorial con Rotación Varimax: Factor 1 Conocimiento: Inatención $(0,91)$, Hiperactividad $(0,85)$ e Impulsividad $(0,67)$. Factor 2 Práctica Pedagógica: Relación Pedagógica $(0,67)$ y Didáctica $(0,94)$. Varianza explicada: Componente 1 Conocimiento: 45,49\%. Componente 2 Práctica Pedagógica: 30,43\%. Total de la varianza explicada: $75,93 \%$.

\section{Procedimiento.}

Revisión de documentos e investigaciones. El estudio se planteó desde la idea de diseñar y validar un instrumento que mida el manejo dado a las Conductas de Hiperactividad y Déficit Atencional de los Escolares.

Diseño y elaboración del instrumento. Teniendo en cuenta la fase anterior se estructuró el instrumento desde las dimensiones de medición agrupando los reactivos en áreas.

Confiabilidad. Después de haber conformado el instrumento, este se aplicó a los docentes de la institución, se procedió al análisis de los reactivos, utilizando el Alfa de Cronbach para determinar su fiabilidad y como descarte de los mismos.

Resultados. Se utilizaron los estadísticos descriptivos de frecuencia y porcentaje, las tablas de contingencia y los mapas perceptuales con el fin de presentar los hallazgos encontrados.
Informe. Se realizó la estructura del documento final en formato consultoría, producto de los hallazgos encontrados en la investigación.

\section{Resultados y discusiones}

En este apartado se describen los métodos estadísticos univariados y multivariados utilizados en los cálculos de fiabilidad, consistencia interna, mapas perceptuales, índices discriminantes y de asociación, y los estadísticos descriptivos, resaltando el coeficiente de Alfa de Cronbach, la correlación de Tau b de Kendall, los Mapas Perceptuales y los Gráficos de Medidas Discriminantes, entre otros.

\section{Estadísticos de fiabilidad y validez de la escala}

\begin{tabular}{l}
$\frac{\text { Tabla 1. Coeficiente de Alfa de Cronbach }}{\text { Estadísticas de fiabilidad }}$ \\
Alfa de Cronbach N de elementos \\
\hline 0,82 \\
\hline
\end{tabular}

El Alfa de Cronbach permite estimar la fiabilidad de un instrumento a través de un conjunto de ítems que se espera que midan el mismo constructo o dimensión teórica (González y Pazmiño, 2015). Al aplicarlo en la escala se puede identificar un coeficiente alto $(0,82)$ basado en las 5 dimensiones (5 elementos).

Tabla 2. Coeficiente de Alfa de Cronbach si se elimina el elemento

\begin{tabular}{ccccc}
\hline $\begin{array}{c}\text { Estadísticas de total de } \\
\text { elemento }\end{array}$ & $\begin{array}{c}\text { Media de escala si } \\
\text { el elemento se ha } \\
\text { suprimido }\end{array}$ & $\begin{array}{c}\text { Varianza de escala } \\
\text { si el elemento se ha } \\
\text { suprimido }\end{array}$ & $\begin{array}{c}\text { Correlación total } \\
\text { de elementos } \\
\text { corregida }\end{array}$ & $\begin{array}{c}\text { Alfa de Cronbach } \\
\text { si el elemento se ha } \\
\text { suprimido }\end{array}$ \\
\hline Inatención & 15,48 & 3,87 & 0,70 & 0,76 \\
Hiperactividad & 15,22 & 4,18 & 0,67 & 0,77 \\
Impulsividad & 15,30 & 3,83 & 0,61 & 0,79 \\
Relación Pedagógica & 15,30 & 4,45 & 0,66 & 0,77 \\
Didáctica & 15,44 & 5,03 & 0,46 & 0,82 \\
\hline
\end{tabular}

De acuerdo con la Tabla 2 se puede identificar que todas las dimensiones son necesarias para la confiabilidad de la escala (Inatención: $\alpha$ 0,76, Hiperactividad: $\alpha$ 0,77, Impulsividad: $\alpha$ 0,79, Relación Pedagógica: $\alpha$ 0,77 y Didáctica: $\alpha$ 0,82).

Tabla 3. Correlación de Spearman

\begin{tabular}{|c|c|c|c|c|c|c|}
\hline \multicolumn{2}{|c|}{ Matriz de Correlación } & \multirow{2}{*}{$\begin{array}{c}\text { Inatención } \\
*\end{array}$} & \multirow{2}{*}{$\begin{array}{c}\text { Hiperactividad } \\
0,69\end{array}$} & \multirow{2}{*}{$\frac{\text { Impulsividad }}{0,64}$} & \multirow{2}{*}{$\begin{array}{c}\begin{array}{c}\text { Relación } \\
\text { Pedagógica }\end{array} \\
0,52\end{array}$} & \multirow{2}{*}{$\frac{\text { Didáctica }}{}$} \\
\hline Inatención & $\begin{array}{l}\text { Coeficiente de } \\
\text { correlación }\end{array}$ & & & & & \\
\hline & Sig. (bilateral) & * & $\mathbf{0 , 0 0}$ & $\mathbf{0 , 0 0}$ & 0,01 & 0,08 \\
\hline \multirow{2}{*}{ Hiperactividad } & $\begin{array}{l}\text { Coeficiente de } \\
\text { correlación }\end{array}$ & 0,69 & * & 0,46 & 0,61 & 0,32 \\
\hline & Sig. (bilateral) & 0,00 & * & 0,02 & 0,00 & 0,10 \\
\hline \multirow[t]{2}{*}{ Impulsividad } & $\begin{array}{l}\text { Coeficiente de } \\
\text { correlación }\end{array}$ & 0,64 & 0,46 & * & 0,52 & 0,44 \\
\hline & Sig. (bilateral) & 0,00 & 0,02 & * & 0,01 & 0,02 \\
\hline \multirow{2}{*}{$\begin{array}{l}\text { Relación } \\
\text { Pedagógica }\end{array}$} & $\begin{array}{l}\text { Coeficiente de } \\
\text { correlación }\end{array}$ & 0,52 & 0,61 & 0,52 & $*$ & 0,57 \\
\hline & Sig. (bilateral) & 0,01 & 0,00 & 0,01 & * & $\mathbf{0 , 0 0}$ \\
\hline \multirow{2}{*}{ Didáctica } & $\begin{array}{l}\text { Coeficiente de } \\
\text { correlación }\end{array}$ & 0,34 & 0,32 & 0,44 & 0,57 & $*$ \\
\hline & Sig. (bilateral) & 0,08 & 0,10 & 0,02 & 0,00 & * \\
\hline
\end{tabular}

El coeficiente de correlación de Spearman mide el grado de asociación entre variables que no siguen una distribución normal, los valores van de -1 a 1 , siendo 0 el valor que indica no correlación, y los signos indican correlación directa e inversa 
(Castro y Ruiz, 2015). La Tabla 3 muestra las correlaciones de Spearman y la Prueba de Significancia Estadística (Sig. Bilateral) arrojando con un $95 \%$ de confianza que existe asociación entre las variables: Inatención e Hiperactividad $(p<0,00)$, Inatención e Impulsividad $(p<0,00)$, Inatención y Relación Pedagógica $(\mathrm{p}<0,01)$, Hiperactividad e Impulsividad $(p<0,02)$, Hiperactividad y Relación Pedagógica $(p<0,00)$, Impulsividad y Relación Pedagógica $(p<0,01)$, Impulsividad y Didáctica $(p<0,02)$ y Relación Pedagógica y Didáctica $(p<0,00)$ evidenciándose así una adecuada consistencia interna entre las dimensiones de la escala.

\section{Mapas perceptuales}

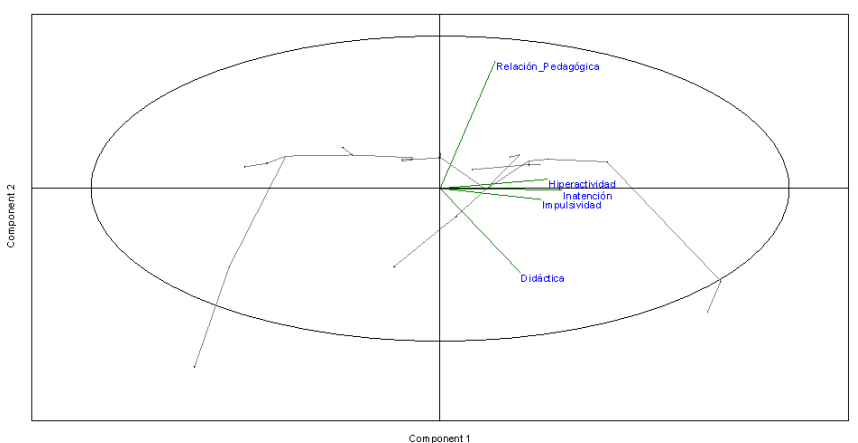

Figura 1. Mapa Perceptual - Conocimiento y Práctica Pedagógica

Para identificar la asociación entre las dimensiones del Conocimiento y la Práctica Pedagógica se utilizó el Análisis de Correspondencias, debido a que es una técnica estadística que se utiliza para analizar, desde un punto de vista gráfico, las relaciones de dependencia e independencia de un conjunto de variables a partir de los datos de una tabla de contingencia (Pérez, Molina y Lechuga, 2013). Para ello asoció a cada una de las modalidades de la tabla, un punto en el espacio Rn (generalmente $n=2$ ) de forma que las relaciones de cercanía/ lejanía entre los puntos calculados reflejen las relaciones de dependencia y semejanza existentes entre ellas. Según la Gráfica 1 se puede percibir que existe relación entre el conocimiento de la Inatención, Hiperactividad e Impulsividad, mientras que las dimensiones de Relación Pedagógica y Didáctica, no se relacionan con ninguna otra dimensión en específico.

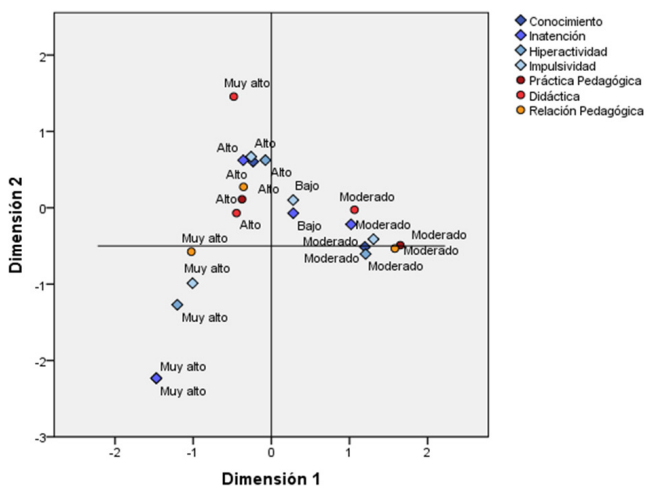

Figura 2. Mapa Perceptual - Conocimiento y Práctica Pedagógica
Tabla 4. Variables transformadas de correlaciones

\begin{tabular}{cccc}
\hline & Relación Pedagógica & Didáctica & Práctica Pedagógica \\
\hline Inatención & $\mathbf{0 , 5 6}$ & 0,40 & 0,44 \\
Hiperactividad & $\mathbf{0 , 5 9}$ & 0,34 & 0,43 \\
Impulsividad & $\mathbf{0 , 6 7}$ & $\mathbf{0 , 5 6}$ & $\mathbf{0 , 5 6}$ \\
Conocimiento & $\mathbf{0 , 5 1}$ & 0,24 & 0,37 \\
\hline
\end{tabular}

Según la Tabla 5 de variables transformadas de correlaciones se puede identificar que la Relación Pedagógica se asocia con la Inatención $(0,56)$, Hiperactividad $(0,59)$, Impulsividad $(0,67)$ y el Conocimiento $(0,51)$, la Didáctica y la Práctica Pedagógica por su parte se asocia con la Impulsividad $(0,67)$.

\section{Estadísticos descriptivos.}

Tabla 5. Tabla de Contingencia - Dimensiones: Conocimiento y Relación Pedagógica

\begin{tabular}{|c|c|c|c|c|c|c|c|c|c|c|c|c|c|}
\hline \multirow{2}{*}{\multicolumn{2}{|c|}{$\begin{array}{l}\text { Dimensiones } \\
\text { Conocimiento }\end{array}$}} & \multicolumn{10}{|c|}{ Relación Pedagógica } & \multirow[b]{2}{*}{ Total } & \multirow[b]{2}{*}{$\%$} \\
\hline & & $\begin{array}{l}\text { Muy } \\
\text { bajo }\end{array}$ & $\%$ & Bajo & $\%$ & Moderado & $\%$ & Alto & $\%$ & $\begin{array}{l}\text { Muy } \\
\text { alto }\end{array}$ & $\%$ & & \\
\hline \multirow{5}{*}{ Inatención } & Muy bajo & 0 & 0 & 0 & 0 & 0 & 0 & 0 & 0 & 0 & 0 & 0 & 0 \\
\hline & Bajo & 0 & 0 & 0 & 0 & 0 & 0 & 1 & 4 & 0 & 0 & 1 & 4 \\
\hline & Moderado & 0 & 0 & 0 & 0 & 5 & 19 & 4 & 15 & 0 & 0 & 9 & 33 \\
\hline & Alto & 0 & 0 & 0 & 0 & 1 & 4 & 11 & 41 & 2 & 7 & 14 & 52 \\
\hline & Muy alto & 0 & 0 & 0 & 0 & 0 & 0 & 2 & 7 & 1 & 4 & 3 & 11 \\
\hline \multirow{5}{*}{ Hiperactividad } & Muy bajo & 0 & 0 & 0 & 0 & 0 & 0 & 0 & 0 & 0 & 0 & 0 & 0 \\
\hline & Bajo & 0 & 0 & 0 & 0 & 0 & 0 & 0 & 0 & 0 & 0 & 0 & 0 \\
\hline & Moderado & 0 & 0 & 0 & 0 & 4 & 15 & 2 & 7 & 0 & 0 & 6 & 22 \\
\hline & Alto & 0 & 0 & 0 & 0 & 2 & 7 & 13 & 48 & 1 & 4 & 16 & 59 \\
\hline & Muy alto & 0 & 0 & 0 & 0 & 0 & 0 & 3 & 11 & 2 & 7 & 5 & 19 \\
\hline \multirow{5}{*}{ Impulsividad } & Muy bajo & 0 & 0 & 0 & 0 & 0 & 0 & 0 & 0 & 0 & 0 & 0 & 0 \\
\hline & Bajo & 0 & 0 & 0 & 0 & 0 & 0 & 1 & 4 & 0 & 0 & 1 & 4 \\
\hline & Moderado & 0 & 0 & 0 & 0 & 5 & 19 & 2 & 7 & 0 & 0 & 7 & 26 \\
\hline & Alto & 0 & 0 & 0 & 0 & 1 & 4 & 10 & 37 & 2 & 7 & 13 & 48 \\
\hline & Muy alto & 0 & 0 & 0 & 0 & 0 & 0 & 5 & 19 & 1 & 4 & 6 & 22 \\
\hline
\end{tabular}

Según la Tabla 6 se puede identificar que el empoderamiento del docente en cuanto a la capacidad que posee para reconocer el cambio del foco atencional por parte de los estudiantes en relación a los estímulos del medio o dirigido a estímulos no relevantes, las faltas cometidas producto del no seguimiento de indicaciones previas, la identificación del exceso de actividad y que no se ajuste a las perspectivas esperadas para la edad, como el traslado del puesto de trabajo, movimientos excesivos o exagerados de partes del cuerpo, correr y hablar en momentos inadecuados, al igual que reacciones predispuestas, agiles e inesperadas ante situaciones que resulten amenazantes, sumado a la carencia de reflexión previa de los mismo y de sus consecuencias, representa una relación directa con el ejercicio de una relación pedagógica mayor con sus estudiantes.

Esto se releja en la calidad de la interacción entre ambas partes dentro del entorno educativo y en el que se incluya aquellos elementos que den apoyo al cumplimiento de las actividades, lo que favorece el desempeño académico al hacer de la enseñanza un proceso más eficiente, que permita el entendimiento, comunicación, evitación de conflictos, aumentando la participación en el aula, el desarrollo de competencia de análisis para la toma de decisiones y una mayor 
inclusión de aquellos estudiantes con alguna característica específica que requiera de un manejo especial.

Por tanto, el mayor conocimiento de dichas características, su manifestación y abordaje, se asocia con una perspectiva de formativa dirigida a los estudiantes, en la que se contribuye a mejorar las relaciones socio-educativas, lo que permite la participación inclusiva y el desarrollo de habilidades que apoyen los procesos de formación.

Tabla 6. Tabla de Contingencia - Dimensiones: Conocimiento y Didáctica

\begin{tabular}{|c|c|c|c|c|c|c|c|c|c|c|c|c|c|}
\hline \multirow{2}{*}{\multicolumn{2}{|c|}{$\begin{array}{l}\text { Dimensiones } \\
\text { Conocimiento }\end{array}$}} & \multirow[b]{2}{*}{$\begin{array}{l}\text { Muy } \\
\text { bajo }\end{array}$} & \multirow[b]{2}{*}{$\%$} & \multirow[b]{2}{*}{ Bajo } & \multicolumn{3}{|c|}{ Didáctica } & \multirow[b]{2}{*}{ Alto } & \multirow[b]{2}{*}{$\%$} & \multirow[b]{2}{*}{$\begin{array}{l}\text { Muy } \\
\text { alto }\end{array}$} & \multirow[b]{2}{*}{$\%$} & \multirow[b]{2}{*}{ Total } & \multirow[b]{2}{*}{$\%$} \\
\hline & & & & & $\%$ & Moderado & $\%$ & & & & & & \\
\hline \multirow{5}{*}{ Inatención } & Muy bajo & & 0 & 0 & 0 & 0 & 0 & 0 & 0 & 0 & 0 & 0 & 0 \\
\hline & Bajo & 0 & 0 & 0 & 0 & 0 & 0 & 1 & 4 & 0 & 0 & 1 & 4 \\
\hline & Moderado & 0 & 0 & 0 & 0 & 5 & 19 & 4 & 15 & 0 & 0 & 9 & 33 \\
\hline & Alto & 0 & 0 & 0 & 0 & 3 & 11 & 10 & 37 & 1 & 4 & 14 & 52 \\
\hline & Muy alto & 0 & 0 & 0 & 0 & 0 & 0 & 3 & 11 & 0 & 0 & 3 & 11 \\
\hline \multirow{5}{*}{ Hiperactividad } & Muy bajo & 0 & 0 & 0 & 0 & 0 & 0 & 0 & 0 & 0 & 0 & 0 & 0 \\
\hline & Bajo & 0 & 0 & 0 & 0 & 0 & 0 & 0 & 0 & 0 & 0 & 0 & 0 \\
\hline & Moderado & 0 & 0 & 0 & 0 & 3 & 11 & 3 & 11 & 0 & 0 & 6 & 22 \\
\hline & Alto & 0 & 0 & 0 & 0 & 5 & 19 & 10 & 37 & 1 & 4 & 16 & 59 \\
\hline & Muy alto & 0 & 0 & 0 & 0 & 0 & 0 & 5 & 19 & 0 & 0 & 5 & 19 \\
\hline \multirow{5}{*}{ Impulsividad } & Muy bajo & 0 & 0 & 0 & 0 & 0 & 0 & 0 & 0 & 0 & 0 & 0 & 0 \\
\hline & Bajo & 0 & 0 & 0 & 0 & 0 & 0 & 1 & 4 & 0 & 0 & 1 & 4 \\
\hline & Moderado & 0 & 0 & 0 & 0 & 5 & 19 & 2 & 7 & 0 & 0 & 7 & 26 \\
\hline & Alto & 0 & 0 & 0 & 0 & 3 & 11 & 9 & 33 & 1 & 4 & 13 & 48 \\
\hline & Muy alto & 0 & 0 & 0 & 0 & 0 & 0 & 6 & 22 & 0 & 0 & 6 & 22 \\
\hline
\end{tabular}

Según la Tabla 7 se puede identificar que entre mayor es la capacidad que posee para reconocer el cambio del foco atencional por parte de los estudiantes en relación a los estímulos del medio o dirigido a estímulos no relevantes, las faltas cometidas producto del no seguimiento de indicaciones previas, la identificación del exceso de actividad y que no se ajuste a las perspectivas esperadas para la edad, como el traslado del puesto de trabajo, movimientos excesivos o exagerados de partes del cuerpo, correr y hablar en momentos inadecuados, al igual que reacciones predispuestas, agiles e inesperadas ante situaciones que resulten amenazantes, sumado a la carencia de reflexión previa de los mismo y de sus consecuencias, representa una relación directa con el ejercicio de una relación pedagógica mayor con sus estudiantes; su manejo de los mismos, mediante estrategias didácticas, será mayor, lo cual se refleja en la búsqueda y uso de técnicas, métodos que le permitan impartir las temáticas dentro del aula, promoviendo la participación activa, ajustando criterios evaluativos, adaptando al estudiante al contexto educativo, y empleando pruebas evaluativas más acordes a las características de estos.

De igual forma, el mayor conocimiento de las características de los alumnos con inatención, hiperactividad de impulsividad, refleja un mejor manejo en el que se promueva el desarrollo de perspectivas académicas y personales, se favorezca el uso de elementos tecnológicos y se contribuya al control de síntomas propios de dichas características.

Tabla 7. Tabla de Contingencia - Conocimiento y Práctica Pedagógica

\begin{tabular}{ccccccc}
\hline Conocimiento & \multicolumn{1}{c}{ Práctica Pedagógica } & \multirow{2}{*}{ Total } & $\%$ \\
& Moderado & $\%$ & Alto & $\%$ & & \\
\hline Moderado & 3 & 11 & 4 & 15 & 7 & 26 \\
Alto & 2 & 7 & 15 & 56 & 17 & 63 \\
Muy alto & 0 & 0 & 3 & 11 & 3 & 11 \\
Total & 5 & 19 & 22 & 81 & 27 & 100 \\
\hline
\end{tabular}

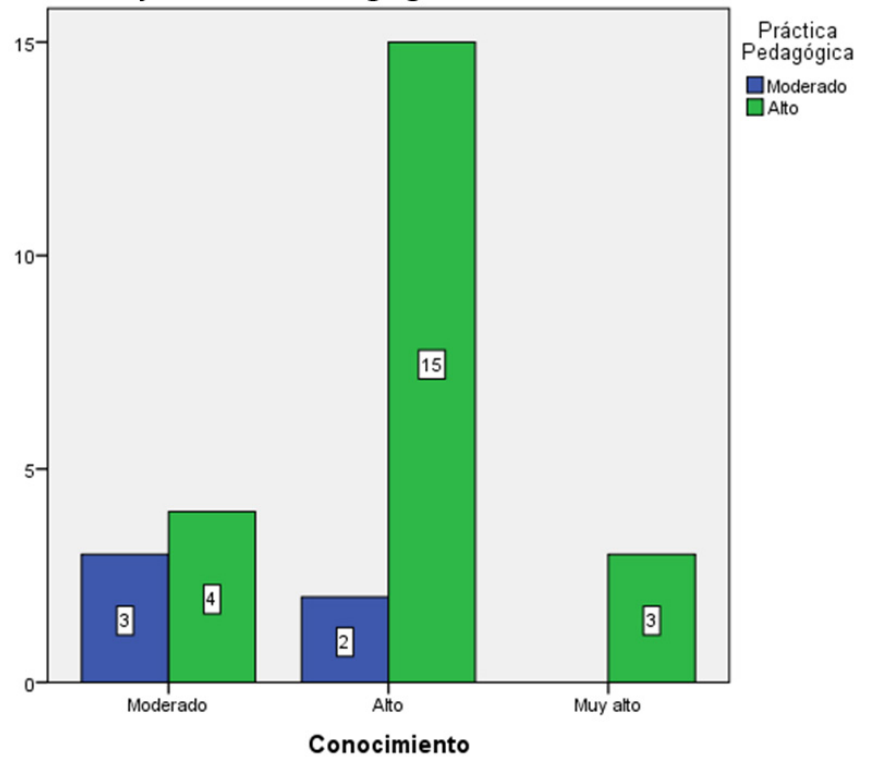

Figura 3. Conocimiento y Práctica Pedagógica

Según la Tabla 8 y la Gráfica 4 se identifican que la relación entre una Práctica Pedagógica alta y un mayor Conocimiento acerca de la Inatención, Hiperactividad e Impulsividad es significativo en cuanto refleja un compromiso con la participación de sus estudiantes, mejoramiento del desempeño académico, formación personal en la integración social y comunitaria del individuo.

Esta tabla a su vez permite evidenciar a su vez que la relación bilateral del conocimiento y la práctica pedagógica representa como los procesos educativos hacen participes a todos los agentes que interactúan en el mismo, sea dentro o fuera del entorno educativo, por lo que el educador realiza acciones que buscan el aporte interrelacionado tanto de las directivas institucionales en sus niveles y ordenamientos funcional, como de padres de familia y estudiantes, en el objetivo de generar espacios de formación más óptimos para las necesidades específicas en especial de aquellos alumnos con algún grado de afectación por en relación a las dimensiones estudiadas.

\section{Discusión}

El panorama es muy complejo si se tiene en cuenta que los docentes deben responder con su escasa preparación en este campo a las situaciones diversas que se presentan en los contextos escolares, especialmente con los pocos recursos disponibles en las zonas rurales y la obligatoriedad de la norma y el derecho que asiste a los colombianos con discapacidades (Orús, Cosculluela y Toledo, 2017). De este modo, la inclusión educativa se posiciona como una tendencia de la actual sociedad, en la que la consigna de hacer partícipes a cada uno de los miembros de la misma, requiere de un conocimiento y reconocimiento de condiciones transitorias o permanentes que potencialmente afectan el aprendizaje (Villa, 2015); las mayores expectativas y demandas sociales por educación 
y especialización para la vida laboral y productiva; y la mayor complejidad de los aprendizajes a adquirir, entre otros (López y Valenzuela, 2015). Por ello, la forma de pensar, las concepciones que los docentes tejen sobre la necesidad de la inclusión educativa y su ejercicio, influyen en las prácticas pedagógicas con las cuales brindan atención a los niños y niñas que presenten algún tipo de discapacidad (Vergara y González, 2017).

En el caso de niños que presentan alteraciones como la hiperactividad, inatención e impulsividad, algunos de sus manifestaciones pueden brindar una correlación que permita un diagnóstico puntual, facilitando su identificación temprana, $\mathrm{y}$ el entendimiento de los comportamientos y afecciones al rendimiento académico que pudieran presentarse. Por tanto, en ocasiones la aglomeración de características comportamentales y de procesamiento cognitivo, se asocian con la presencia del trastorno déficit de atención, sea este acompañado de hiperactividad e impulsividad, y del cual se refiere que aunque se carecen de estudios concluyente para determinar su origen (Estévez y Guerrero, 2017), y frente a lo cual se realiza una evaluación diagnóstica basada en la observación de las conductas manifiestas, sin descartar por ello una base biológica de los mismos, lo que indica que se trate de un trastorno intrínseco a la persona que lo padece que, en opinión de diferentes expertos, se debe a un retraso en el desarrollo neuropsicológico (Miranda, 2004; Barkley, 2006; Lavigne y Romero, 2010; Orjales, 2011 citado por Gómez, Soria, Rico y Carrasco, 2017).

En búsqueda de un mayor entendimiento del sistema social que interviene en la educación como proceso, se resalta el concepto de sociología la educación, expuesto por Bourdieu, en el que menciona que la finalidad de esta es estudiar la reproducción cultural y la reproducción social (Guerrero, 2015), para lo cual las instituciones educativas poseen una autonomía que dota al docente de una autoridad para ejercer la acción educativa pedagógica; por lo que se asume que la formación docente debe contar con sustentos teóricos y explicativos de la diversidad de conductas y situaciones que se presentan dentro de las aulas, en especial, con niños cuyas características de inatención, hiperactividad e impulsividad, representan una barrera que dificulta la facilidad de adquisición de conocimientos y cumplimiento de objetivos, es por ello que el docente necesita estar formado para trabajar con niños con TDAH (Daniel, Gupta y Sagar, 2013).

Dicho esto, al observar las puntuaciones y analizar desde los aspectos teóricos planteados, se puede identificar en un contraste con estudios previos acerca de los conocimientos y creencias que poseen los docentes frente a la inatención, hiperactividad e impulsividad, en especial entendiéndolas como un conjunto diagnóstico, tal como representa el TDAH, su conocimientos frente al tema fueron relativamente escasos: $32.2 \%$ señalaron que el TDAH se debe a vulnerabilidades biológicas y causas genéticas, y $65.5 \%$ contestaron que es consecuencia de una mala crianza. El $67.8 \%$ reconoció que los niños con TDAH experimentan disfunción social, además de la académica (Nur, 2010 citado por Palacios, et. al. 2013).

De este modo, los docentes consideran que el manejo referido a los estudiantes con algún tipo de afectación anteriormente mencionada debe ser de tipo conductual, y es el que emplean como herramienta de mitigación de este tipo de conductas al tiempo que buscan favorecer los procesos educativos (Fainholc, Nervi, Romero y Halal, 2015). De igual forma, este tipo de acciones corresponden de manera directa al tipo de conocimientos que posea el docente frente a la temática, lo que se refleja en el manejo impartido y el direccionamiento profesional en cuanto a tratamiento pedagógico como soporte del manejo externo enfocado directamente a dicha alteración (Fernández, 2016).

Es por ello que reconocer una estructura compleja, ecológica, con múltiples intereses y posiciones dentro de las relaciones de poder es útil para adoptar posiciones más pertinentes y realistas sobre la educación desde un enfoque para la promoción de la salud y sobre todo para la vida en contexto en términos de las implicaciones metodológicas que sean útiles a este fin, haciendo uso de actividades y estrategias didácticas. Esta situación implica comprender que una concepción de educación para la salud centrada en el desarrollo del ser humano no desde la perspectiva patológica, sino desde la perspectiva del potencial y el desarrollo de competencias, es fundamental para ejercer un apoyo a los procesos de vida.

En el anterior contexto y obedeciendo al fenómeno de estudio los docentes presentan actitudes más favorables hacia el acompañamiento, manejo y atención de conductas que pueden ser consideradas disruptivas en el aula y que implican generar manejos diferenciales en lo relacionado al componente didáctico y metodológico en el aula, lo que contrasta con estudios previos en los que las manifestaciones conductuales de los docentes frente a estas conductas del alumnado oscilan entre respuestas apropiadas y creativas a los requerimientos de cada estudiante, al tiempo que subrayan el factor afectivo que marca sus acciones. (Portillo y Wiens, 2013).

El recurso cognitivo de los docentes, en términos de conocimientos sobre el manejo de la inatención, impulsividad e hiperactividad, les permite a los docentes tomar actividades más positivas hacia los estudiantes con esta connotación, es decir que los docentes se consideran con la capacidad para estimular el aprendizaje y adquirir conocimientos nuevos. Este elemento constituye uno de los factores más importantes para proporcionar buenas prácticas educativas más incluyentes y más pertinentes.

La observación consistente de los resultados permite determinar que los docentes participantes, manejan un lenguaje y una construcción conceptual sobre la inatención, impulsividad e hiperactividad. Lo cual evidencia la presencia de docentes con una base conceptual frente a las temáticas, que a su vez podría asociarse con el discurso y formulación de estrategias educativas desde la organización administrativa y académica de la institución, constituyéndose como un factor crucial en 
las labores de apoyo y asesoramiento al cuerpo docente en las aulas (Armijos, 2017).

Por último, se hace referencia a la motivación implícita de cada uno de los docentes de la institución educativa, quienes sin duda disponen de una actitud favorable, al instruirse en el reconocimiento de conductas de interés al interior de las aulas de clase, lo cual constituye un valor agregado en el marco de la atención a la diversidad y al mejoramiento de la relación pedagógica, la cual busca la indagación y pensamiento propios, yendo más allá de lo visible y actuando conscientemente en la búsqueda de nuevos conocimientos; por lo que se trata de una posición en la enseñanza abierta a las ocasiones y a los acontecimientos que se presentan, próxima a una pedagogía del evento (Atkinson, 2011; Correa, 2015) que sin duda se puede asociar al nivel de compromiso del equipo directivo en la búsqueda de una educación pertinente de calidad e inclusiva.

\section{Conclusiones}

A partir del estudio realizado, se determina que en la dimensión de inatención, refleja la capacidad que posee el docente para reconocer las dificultades para movilizar o cambiar de foco atencional frente a estímulos externos por parte de los estudiantes en situaciones que requieren atender a los detalles, los errores cometidos por descuido en el desarrollo de las tareas, las dificultades para mantener la atención, la escucha, el seguimiento de instrucciones y la finalización de tareas, la organización de las tareas y actividades, la evitación, molestia o disgusto hacia las tareas que requieren un esfuerzo mental sostenido, la perdida de objetos necesarios para el desarrollo de actividades o tareas, y la fijación de la atención a estímulos irrelevantes.

Los problemas atencionales son un elemento evolutivo que cuyo desarrollo depende de la edad y que se va consolidando con la edad hasta la adolescencia (López y García, 2004; Servera y Galván, 2001 citados por Castellano, 2015) y cuyas causas obedecen a factores endógenos y exógenos, que determinan el nivel de atención que puede prestarse a los estímulos del medio; de igual forma, existen ciertos factores que determinan la aparición de problemas atencionales, tales como problemas orgánicos, características de las tareas asignadas, estados transitorios, entre otros.

En cuanto a la dimensión de hiperactividad, esta registra de qué manera el docente logra reconocer comportamientos caracterizados por la actividad excesiva y fuera de lo normal por parte de los estudiantes como sacudir en exceso las manos o los pies, moverse reiteradamente, abandonar el asiento en el aula de clase en situaciones en que se espera que permanezca sentado, correr o saltar excesivamente en situaciones en las que es innecesario hacerlo o hablar en exceso durante el desarrollo de actividades o tareas, lo que resume la premisa de que los niños hiperactivos basan su conducta en tres aspectos: es desatento, nivel de actividad elevado y una falta de control excesiva (Gutiérrez, 2014).

Entre tanto, en lo referente a la impulsividad, esta nos permite identificar la capacidad que posee el docente en el reconocimiento de la predisposición a reaccionar de forma inesperada, rápida, y desmedida ante una situación externa que puede resultar amenazante, o ante un estímulo interno propio del individuo, sin tener una reflexión previa ni tomar en cuenta las consecuencias que pueden provocar sus actos en los estudiantes como precipitar la respuesta antes de terminar la pregunta, las dificultades para esperar su turno, interrumpir o inmiscuirse en actividades de otros; por lo que para poder ayudar a los niños impulsivos a pensar y organizar su pensamiento, ellos requerían verbalizar, en voz alta, sus pensamientos, (Meichenbaum y Orjales, 2003; Malagón, Giraldo y Posada, 2016) para de este modo lograr ordenas los pensamientos en una serie de pasos o auto-instrucciones y de este modo dar solución a las diversas situaciones que se presenten.

Por su parte, la relación pedagógica comprende las interacciones entre el docente, el contenido y el estudiante, en un escenario educativo donde se propicia la formación del ser humano teniendo en cuenta los materiales de apoyo para la realizar actividades y facilitar la comprensión del tema, el espacio en el aula para visualizar las actividades semanales, la comunicación con los estudiantes, la asignación del tiempo para la entrega de las actividades o tareas, el evitar señalar a un estudiante o apartarlo del grupo durante el desarrollo de una clase, el impedir por parte de los compañeros conductas de rechazo hacía un estudiante durante el desarrollo de actividades o tareas, la generación de espacios de comunicación en el aula explicando cómo identificar y corregir errores, proporcionar el tiempo necesario para el desarrollo de las actividades o tareas. Dicha interacción se fundamenta en el diálogo y el acompañamiento, que permite al mismo tiempo una interacción e intercambio mutuo, (Correa, 2015) que a su vez incluye un aspecto reflexivo y colaborativo mutuo.

En cuanto al contenido didáctico implementado por los docentes, se indican aquellas habilidades del educador para la búsqueda de métodos y técnicas para mejorar la enseñanza, favoreciendo la inclusión en el aula en las cuales se incluyen los criterios de evaluación de acuerdo con las necesidades de cada estudiante, los tipos de prueba acordes a las características de los estudiantes, la atención individualizada en el aula, las actividades en el aula para facilitar la adaptación de los estudiantes, la utilización de medios audiovisuales para focalizar la atención, las instrucciones de forma corta y clara, el establecimiento de las expectativas de aprendizaje y de comportamiento. Esta temática incluye saberes teóricos disponibles, valores y saberes experienciales de los propios docentes (Davini, 2015).

Concluyendo con lo anterior, son diversos los puntos que resaltan del estudio realizado, en los que se resaltan premisas referentes tanto a la hiperactividad, impulsividad e inatención, las cuales son consideradas síntomas primarios 
del trastorno, y suelen ir acompañadas por dificultades cognitivas, emocionales, académicas e interpersonales que, a su vez, se constituyen en síntomas secundarios del desorden (Korzaniowsk y Ison, 2013 citados por Malagón, Giraldo y Posada, 2016). Este criterio frente a características singulares que pueden presentar los estudiantes, son consideradas como una temática que en ocasiones tiende a dejarse a un lado, o se limita a un conocimiento superficial del mismo, por lo que para muchos docentes sigue siendo un problema desconocido, lo que dificulta el diagnóstico y por lo tanto retrasa las herramientas de tratamiento (Daniel, Gupta y Sagar, 2013).

Es a partir de este conocimiento que se fortalecen las relaciones pedagógicas que posteriormente dan paso al uso de prácticas pedagógicas enfocadas en la inclusión, adaptación, desarrollo y formación de los estudiantes en las aulas, tal como se evidenció en los resultados obtenidos, los cuales demuestran que al existir una mayor presencia de conocimientos frente al tema, los docentes se encuentran más capacitados para la formulación, indagación y puesta en marcha de estrategias pedagógicas que permitan al estudiante formar habilidades para su desempeño dentro y fuera de las aulas.

\section{Referencias}

Acosta Rodríguez, V. M. (2016). Lenguaje e inclusión educativa: reflexiones para atenuar el fracaso escolar.

Aguiar, A.P. Kieling, R.R. Costa, A.C. Chardosim, N. Dorneles, B.V. Almeida, M.R. Mazzuca, A.C. Kieling, C. Rohde, L.A. (2014). Increasing teachers' knowledge about ADHD and learning disorders: an investigation on the role of a psychoeducational intervention. J Atten Disord. 18(8):691-8. doi: 10.1177/1087054712453171.

Alvárez Menéndez, S. y Pinel González, A. (2015). Trastorno por déficit de atención con hiperactividad en mi aula de infantil. Revista Española de Orientación y Psicopedagogía, 26 (3), 141-152.

Angarita-Ortiz, M. F., Forgiony-Santos, J. y Rivera-Porras, D. (2018). Rol y futuros retos del psicólogo en el campo de la psicología educativa en Colombia. En CarrilloSierra, S. M., Sanabria-Herrera, B. Bermúdez-Pirela, V. y Espinosa-Castro, J. F. (Ed.). Actores en la educación: Una mirada desde la psicología educativa (pp. 17-45). Barranquilla, Colombia: Ediciones Universidad Simón Bolívar. ISBN: 978-958-5533-40-0. Recuperado de: http://bonga.unisimon.edu.co/handle/123456789/2490.

Aperador Chasoy, A. M., Díaz Pérez, E. E. y Orozco Gómez, S. M. (2015). Estrategias para fortalecer las competencias de los docentes, que llevan a cabo procesos de inclusión educativa en dos colegios oficiales de Bogotá (Master's thesis, Universidad de La Sabana).

Arenas-Villamizar, V. V., Silva-Monsalve, G. I., AnnicchiáricoLobo, J. H., Contreras-Manrique, L., Luna-Rodríguez, G. R. y Raynaud, N. (2018). Tendencias en intervención psicológica y psicoterapia en el Norte de Santander-
Colombia. Archivos Venezolanos de Farmacología y Terapéutica, 37(5), 546-551. Recuperado de: http://www.revistaavft.com/images/revistas/2018/ avft_5_2018/19tendencias_intervenci\%C3\%B3n.pdf.

Armijos Cabrera, O. D. (2017). Estrategias psicopedagógicas dirigidas a docentes y alumnos que excluyen a niños y niñas con problemas de atención.

Atkinson, D. (2011). Art, Equality and Learning. Pedagogies against the State. Rotterdam: Sense.

Bazán, D. y González, L. (2017). Pedagogía de la formación docente: modelos, relevancia y acuerdos. Paulo Freire, (6), 65-80.

Bonilla-Cruz, N. J., Forgiony-Santos, J., Hernández-Lalinde, J., Carrillo-Sierra, S. M., Rivera-Porras, D., NuvánHurtado, I. L. y Martínez-Santana, M. C. (2018). Apoyo social percibido en el abordaje terapéutico de adolescentes con orientación suicida. Archivos Venezolanos de Farmacología y Terapéutica, 37(5), 518-522. Recuperado de: http://www.revistaavft.com/images/revistas/2018/ avft_5_2018/14apoyo_social_percibido_abordaje.pdf.

Bonilla-Cruz, N. J., Villamizar-Bermúdez, Z. y ArenasVillamizar, V. V. (2017). Estrategias de afrontamiento y orientación suicida en el post-aborto en mujeres de El CAM-Cúcuta. En Albornoz-Arias, N., Mazuera-Arias, R. y Espinosa-Castro, J. F., Adolescencia: Vulnerabilidades. Una mirada interdisciplinaria (pp. 253-281). Barranquilla: Ediciones Universidad Simón Bolívar recuperado de: http://bonga.unisimon.edu.co/handle/123456789/2331.

Calderón López Velarde, J. (2016). ¿Educación o aprendizaje a lo largo de la vida?. Revista Interamericana de Educación de Adultos, 38 (1)

Calvo, G., Lara, D. B. R. y García, L. I. R. (2017). Un diagnóstico de la formación docente en Colombia. Revista Colombiana de Educación, (47).

Cantor-Silva, M. I., Pérez-Suarez, E. y Carrillo-Sierra, S. M. (2018). Redes sociales e identidad social. AiBi revista de investigación, administración e ingeniería, 6(1), 7482. Recuperado de: http://revistas.udes.edu.co/site/index. php/AiBi/article/view/477/pdf_1.

Carrillo-Sierra, S. M., Rivera-Porras, D., Forgiony-Santos, J., Nuván-Hurtado, I. L., Bonilla-Cruz, N. J. y ArenasVillamizar, V. V. (2018). Habilidades para la vida como elementos en la promoción y educación para la salud en los adolescentes y jóvenes. Archivos Venezolanos de Farmacología y Terapéutica, 37(5), 567-572. Recuperado de: http:/www.revistaavft.com/images/revistas/2018/ avft_5_2018/22habilidades_vida_elementos_elementos. pdf.

Carrillo-Sierra, S. M., Sanabria-Herrera, B. BermúdezPirela, V. y Espinosa-Castro, J. F. (2018). Actores en la educación: Una mirada desde la psicología educativa. Barranquilla, Colombia: Ediciones Universidad Simón Bolívar. ISBN: 978-958-5533-40-0. Recuperado de: http://bonga.unisimon.edu.co/handle/123456789/2490. 
Castellano Bermúdez, M. J. (2015). Intervención en el aula para la mejora de la atención y el rendimiento en el alumnado de segundo nivel de educación primaria: eficacia de las auto-instrucciones y de la auto-observación.

Castro, A. M. P. y Ruiz, M. L. B. (2015). Estandarización de las pruebas HOTV, Snellen y ETDRS en niños de 5 a 15 años de edad dentro del protocolo REISVO: una prueba piloto. Ciencia y Tecnología para la Salud Visual y Ocular, 13(2), 11-28.

Cauas, D. (2015). Definición de las variables, enfoque y tipo de investigación. Bogotá: biblioteca electrónica de la universidad Nacional de Colombia.

Centro de Estudios Educativos y Servicios Integrales de Evaluación y Medición Educativa (SIEME) y Heurística Educativa, S. (2013). Estándares para la educación básica. Experiencia de mejora continua en escuelas mexicanas del nivel básico, con base en estándares curriculares, de desempeño docente y gestión escolar. Revista Latinoamericana de Estudios Educativos (México), XLIII (3), 23-72.

Correa, J. M. (2015). Com aprens a ser mestra?: TIC, gènere i narratives visuals de futures mestres d'educació infantil. REIRE Revista d'Innovació i Recerca en Educació, 8(2), 256-268.

Costa, L. S., Barros, V. F., Lopes, M. C. y Marques, L. P. (2015). La formación docente y la educación de jóvenes y adultos: análisis de la práctica pedagógica para la enseñanza de ciencias. Formación universitaria, 8(1), 0312.

Daniel LT, Gupta S, Sagar R, (2013). Effect of educational module on knowledge of primary school teachers regarding early symptoms of childhood psychiatric disorders. Indian J Psychol Med [serial online] 2013 [cited 2017 Oct 30]; 35:368-72.

Davini, M. C. (2015). La formación en la práctica docente. Buenos Aires: Paidós.

De Tezanos, A. (2015). Oficio de enseñar-saber pedagógico: la relación fundante. Revista Educación y Ciudad, (12), $7-26$.

Delors, J. (2013). Los cuatro pilares de la educación. Galileo, (23).

Duque-Moncada, Y. A., Quintero-Sánchez, C. A., RemolinaCorredor, B. I., Carrillo-Sierra, S. M. y Rivera-Porras, D. (2018). Desempeño y manejo terapéutico de las funciones ejecutivas complejas en estudiantes de 11 grado de una institución de educación pública. Archivos Venezolanos de Farmacología y Terapéutica, 37(5), 534-538. Recuperado de: http://www.revistaavft.com/ images/revistas/2018/avft_5_2018/17desempeno_y_ terap $\% \mathrm{C} 3 \%$ A9utico.pdf.

Echeverry, O. L. (2017). Hacia una historia de la práctica pedagógica colombiana. Revista Colombiana de Educación, (4).
Estévez, B. E. (2015). La inclusión educativa del alumnado con tda/tdah. Rompiendo con las barreras curriculares y organizativas en los centros escolares de educación primaria (Doctoral dissertation, Universidad de Granada).

Estévez, B. E. y Guerrero, M. J. L. (2017). Inclusión educativa del alumnado con TDA/H: estrategias didácticas generales y organizativas de aula. Revista de Educación Inclusiva, 8(3).

Estévez, B. y León, M. J. (2014). Una mirada inclusiva hacia el tdah en las aulas escolares. In XI Congreso Internacional y XXXI Jornadas de Universidades y Educación Inclusiva.

Fainholc, B., Nervi, H., Romero, R. y Halal, C. (2015). La formación del profesorado y el uso pedagógico de las TIC. Revista de Educación a Distancia, (38).

Fernández, J. (2016). Una alternativa al fracaso escolar: Hablemos de buenas prácticas. Psicología, Conocimiento y Sociedad, 6(1), 254-262.

Forgiony-Santos, J. (2019). Modelos pedagógicos: Concepciones, prácticas contexto. Revista AiBi, 7(1), 3540. Recuperado de: http://revistas.udes.edu.co/site/index. php/aibi/article/view/498.

García Barrera, J. P. (2016). Análisis estadístico exploratorio del proyecto" la nueva aurora" del ministerio de la vivienda, miduvi (Bachelor's thesis, Espol).

Garzón, L. P. (2017). La observación de la práctica pedagógica en la formación de futuros docentes. Pedagogía y saberes, (24), 27-31.

Gómez, C. G., Soria, I. N., Rico, A. G. y Carrasco, F. F. (2017). Estrategias de optimización de alumnado con trastorno por déficit de atención e hiperactividad TDA-H. International Journal of Developmental and Educational Psychology. Revista INFAD de Psicología., 7(1), 85-94.

González Alonso, J. y Pazmiño Santacruz, M. (2015). Cálculo e interpretación del Alfa de Cronbach para el caso de validación de la consistencia interna de un cuestionario, con dos posibles escalas tipo Likert. Revista Publicando, 2(1), 62-67.

González-Gómez, D. y Carrillo-Sierra, S. M. (2018). Inteligencia emocional en docentes de primaria. En Carrillo-Sierra, S. M., Sanabria-Herrera, B. BermúdezPirela, V. y Espinosa-Castro, J. F. (Ed.). Actores en la educación: Una mirada desde la psicología educativa (pp. 132-157). Barranquilla, Colombia: Ediciones Universidad Simón Bolívar. ISBN: 978-958-553340-0. Recuperado de: http://bonga.unisimon.edu.co/ handle/123456789/2490.

Guerrero, A. (2015). Manual de sociología de la educación

Gutiérrez, D. A. M. (2014). El niño hiperactivo: rol del docente en su proceso de adaptación en la educación primaria (Doctoral dissertation, 99)

Henao, B. L., Isaza, L. S. y Gómez, M. E. (2017). Hacia la construcción de horizontes alternativos para la práctica pedagógica. Pedagogía y Saberes, (21), 89-98. 
Hurtado-Olaya, P., García-Echeverri, M., Rivera-Porras, D. y Forgiony-Santos, J. (2018). Las estrategias de aprendizaje y la creatividad: Una relación que favorece el procesamiento de la información. Revista Espacios, 39 (17), 12-29. Recuperado de: http://www.revistaespacios. com/a18v39n17/18391712.html.

López, S. I. M. y Valenzuela, B. G. E. (2015). Niños y adolescentes con necesidades educativas especiales. Revista Médica Clínica Las Condes, 26(1), 42-51.

López-Roldán, P. y Fachelli, S. (2015). Metodología de la investigación social cuantitativa. Bellaterra (Cerdanyola del Vallès): Dipòsit Digital de Documents, Universitat Auònoma de Barcelona.

Lucio, R. (2017). Educación y pedagogía, enseñanza y didáctica: diferencias y relaciones. Revista Universidad de la Salle, (17), 35-46.

Malagón, C. N., Giraldo, J. C. y Posada, N. H. (2016). Estrategias neuropsicopedagógicas para niños y niñas con bajo rendimiento académico asociado a TDAH. ENClave Social, 4(2).

Méndez, R. V. M., Coronado, E. J. D., Monroy, J. C. y Arriaga, X. J. (2017). El método estadístico de análisis discriminante como herramienta de interpretación del estudio de adicción al móvil, realizado a los alumnos de la Licenciatura en Informática Administrativa del Centro Universitario UAEM Temascaltepec. RIDE Revista Iberoamericana para la Investigación y el Desarrollo Educativo, 7(14), 222-247.

Montánchez-Torres, M. L., Jornet-Meliá, J. M., PeralesMontolío, M. J., Carrillo-Sierra, S. M. y Wilches-Durán, S. Y. (2017). Educación inclusiva. Estado Zulia: Ediciones Universidad del Zulia. ISBN 978-980-402-214-9. Recuperado de: http://bonga.unisimon.edu.co/bitstream/ handle/ $123456789 / 2104 /$ Educaci $\%$ C3\%B3n\%20 inclusiva.pdf? sequence $=3$ yisAllowed $=y$.

Moreno, E. A. R. (2017). Concepciones de práctica pedagógica. Revista Folios, (16), 105-129.

Nur N, Kavakc O. Elementary school teachers' knowledge and attitudes related to attention deficit hyperactivity disorder. HealthMED 2010; 4 (2):350-355.

Orús, M. L., Cosculluela, C. L. y Toledo, S. V. (2017). Habilidades sociales de niños con déficits atencionales $\mathrm{y}$ contextos escolares inclusivos//Social skills of children with attentional deficits and inclusive school settings. REOP-Revista Española de Orientación y Psicopedagogía, 28(2), 113-121.

Palacios-Cruz et al. (2013). Conocimientos y creencias sobre el trastorno por déficit de atención con hiperactividad en maestros de tres países latinoamericanos. Salud Mental $2013 ; 36: 285-290$

Palomares Ruiz, A. (2017). Retos de la educación inclusiva para construir una sociedad incluyente.
Pérez, M. B., Molina, R. T. y Lechuga, E. N. (2013). Métodos cuantitativos 4 ed. Herramientas para la investigación en salud. Universidad del Norte.

Portillo, P. y Wiens, T. (2013). "TDAH: conocimientos, praxis y actitud de los docentes de la EEB”. Eureka (Asunción) en Línea, 10(1), 28-39.

Preiss, David D, Calcagni, Elisa, Espinoza, Ana María, Gómez, Daniela, Grau, Valeska, Guzmán, Valentina, Müller, Magdalena, Ramírez, Francisca y Volante, Paulo. (2014). Buenas Prácticas Pedagógicas Observadas en el Aula de Segundo Ciclo Básico en Chile. Psykhe (Santiago), 23(2), 1-12.

Rivera-Porras, D., Gallardo-Pérez, H. J., Mora-Ortiz A. R., Gómez-Machado, L. D. y Barrios-Barrios, D. M. (2018). Rendimiento académico y relaciones intrafamiliares en estudiantes repitentes de secundaria. En CarrilloSierra, S. M., Sanabria-Herrera, B. Bermúdez-Pirela, V. y Espinosa-Castro, J. F. (Ed.). Actores en la educación: Una mirada desde la psicología educativa (pp.185-210). Barranquilla, Colombia: Ediciones Universidad Simón Bolívar. ISBN: 978-958-5533-40-0. Recuperado de: http://bonga.unisimon.edu.co/handle/123456789/2490.

Rivero, D. (2013). Metodología de la Investigación.

Torres, A. L. R. (2015). Subjetividades docentes en la universidad pública colombiana. Comunidades de práctica a propósito de sus narraciones. Revista Colombiana de Educación, (68), 229-263.

Vergara, M. G. y González, J. C. (2017). Vivencias de practicantes de pedagogías en ciencias: prácticas de conocimiento científico y pedagógico. Educação e Pesquisa, 43(2), 341-356.

Villa, M. L. M. (2015). Concepciones docentes para responder a la diversidad: ¿ Barreras o recursos para la inclusión educativa?/Teacher's conceptions to respond to diversity: Barriers or resources for inclusive education?. Psicoperspectivas, 14(3), 68-79.

Villamizar-Novoa, M. J., Rodríguez-Viloria, K. D., CarrilloSierra, S. M. y Torrado, J. L. (2018). Memoria de trabajo en estudiantes de once grados de una institución educativa de Cúcuta. En Carrillo-Sierra, S. M., Sanabria-Herrera, B. Bermúdez-Pirela, V. y Espinosa-Castro, J. F. (Ed.). Actores en la educación: Una mirada desde la psicología educativa (pp. 211-231). Barranquilla, Colombia: Ediciones Universidad Simón Bolívar. ISBN: 978-9585533-40-0. Recuperado de: http://bonga.unisimon.edu. co/handle/123456789/2490. 